\title{
A Dosimetric Comparison between RapidArc and Conventional 3D Conformal Radiotherapy in Post-Operative Irradiation of Low Grade Gliomas: Any Added Benefit?
}

\author{
RADWA H. AZAB, M.Sc.*; KARIM MASHHOUR, M.D.*; MOHAMED ABDALLA, M.D.*; \\ HANAN DARWISH, M.D.*; HODA ABD EL-GHANY, M.Sc.** and SHAWKY EL-HADDAD, F.R.C.R., M.D.*
}

The Department of Clinical Oncology, Faculty of Medicine* and The Department of Medical Physics, Kasr Al-Ainy Hospital**, Cairo University, Egypt

\begin{abstract}
Background: Low-Grade Gliomas (LGG) comprise a rare group of central nervous system tumors and clinically challenging cases to manage. They account for approximately $15 \%$ of all primary brain neoplasms. Radiotherapy (RT) remains an important component in the treatment of low grade gliomas and Three-Dimensional Conformal RT (3DCRT) is the current state of art for RT delivery. The main gain of modern RT technology is more likely reducing treatment related toxicity rather than an improvement in overall survival. RapidArc (RA) technology (Varian Medical Systems, Palo Alto, CA) is a novel radiation therapy technique integrating 3 important factors for treatment delivery.
\end{abstract}

Aim of the Study: The aim of the study is to investigate the potential dosimetric benefit of RapidArc (RA) in comparison to conventional 3D-CRT for low grade gliomas of the brain regarding target coverage and doses received by organs at risk (OAR).

Material and Methods: Twenty patients diagnosed with low grade glioma (WHO grade I-II) were referred to our center for postoperative irradiation using 3D-CRT. The prescribed dose was $54 \mathrm{~Gy} / 30 \mathrm{~F} / 6$ weeks. CT scans of the 20 patients was done and delineation of the target volumes and organs at risk was performed. Two set of plans were done for each patient, one using conventional 3D-CRT and other using a RA plan. Dosimetric parameters regarding target coverage and dose received by OAR were evaluated and compared. Monitor units was also calculated.

Results: Target coverage in terms of $\mathrm{V} 95 \%$ was significantly better in the RA plans with a value of $98.0 \pm 1.9$ versus $96.0 \pm 2.6$ for the 3D-CRT plans ( $p$-value of 0.006 ). The RA plans gave a better conformity with a CI95\% of $1.01 \pm 0.012$ compared to1.07 \pm 0.024 achieved with the $3 \mathrm{D}$-CRT plans ( $p$ value of 0.678 ). The Homogeneity Index (HI) was higher for the 3D-CRT plans with a value of $0.131 \pm 0.112$ compared to $0.097 \pm 0.033$ for the RA plans ( $p$-value of 0.114 ). The OAR received less dose in RA than 3D-CRT except for both lenses which received higher doses in the RA plans with a significant

Correspondence to: Dr. Radwa H. Azab, The Department of Clinical Oncology, Faculty of Medicine, Cairo University, Egypt $p$-value of 0.002 and 0.001 for the right and left lens, respectively. The maximum dose and D1\% for the healthy brain tissue was significantly lower in the RA plans when compared to 3D-CRT ( $p$-value of 0.001 ). With respect to the average MUs \pm SD needed per fraction, it was found to be $257.6 \pm 16.1$ for the 3D-CRT plans as opposed to 355.6 $\pm 44.4 \mathrm{RA}$ plans ( $p$ value of 0.001).

Conclusion: RA plans dosimetrically achieved a better PTV coverage, dose conformity, more homogenous dose distribution and better OAR sparing when compared to conventional 3D-CRT plans except for both lenses. The 3D-CRT plans utilized a lower number of MUs than RA.

Key Words: 3D-CRT-Rapid arc - Low grade gliomas Dosimetric.

\section{Introduction}

LOW-Grade Gliomas (LGG) comprise a rare group of central nervous system tumors and clinically challenging cases to manage. They account for approximately $15 \%$ of all primary brain neoplasms $[1,2]$. Surgery remains the cornerstone in the treatment of low grade gliomas [3].

The European Organization for Research and Treatment of Cancer (EORTC) 22845 is a large phase III trial including patients with a pathological diagnosis of low grade gliomas comparing early administration of post-operative radiotherapy versus delaying the treatment until the time of disease progression. The results of the study demonstrated that early post-operative irradiation increases the PFS (5.3 vs 3.4 years) when compared to the deferred group. However, OS was similar between both groups [4].

Radiotherapy (RT) remains an important component in the treatment of low grade gliomas and three-dimensional conformal RT (3DCRT) is the 
current state of art for RT delivery. The main gain of modern RT technology is more likely reducing treatment related toxicity rather than an improvement in overall survival [5]. Radiation therapy treatment has shifted and evolved from threedimensional conformal radiotherapy (3D-CRT) to Intensity-Modulated Radiotherapy (IMRT). The main drawbacks of IMRT are the longer time of the treatment planning process and the need of a complex physics quality assurance. In addition, IMRT uses a larger number of Monitor Units (MUs) and multiple fixed-angle beams, which lengthens the time of treatment delivery exposing the patient to low-dose irradiation $[6,7]$.

RapidArc (RA) technology (Varian Medical Systems, Palo Alto, CA) is a novel radiation therapy technique integrating 3 important factors for treatment delivery; simultaneous variation of the Multileaf Collimator (MLC) position while having a continuous arc motion of the gantry, gantry speed and dose rate [8-10] with the ability to achieve highly conformal plans, less MU and shorter delivery times when compared to IMRT $[11,12]$.

\section{Material and Methods}

An approval from our Ethical and Institutional Scientific Committees was obtained on the study design.

\section{1- Patient selection and eligibility:}

Twenty patients with a pathological diagnosis of low grade gliomas (WHO grade I-II) were referred to our Center of Clinical Oncology and Nuclear Medicine (NEMROCK), Cairo University for post-operative irradiation using 3D-CRT technique during the period June 2014 till July 2016. A RA plan was done for each patient and compared to 3D-CRT. Table (1) shows patients demographic data tumor characteristics.

\section{2- Patient preparation:}

A dedicated planning Computed Tomography (CT) scan (General Electric, USA) was performed for each patient. Patients were aligned in supine position with their head placed in a neutral to flexed position using a Duncan headrest B. Immobilization was achieved by using an Aquaplast facemask (WFR Aquaplast, orfit, NJ). All patients were scanned from skull vertex to the chin with the C.T cuts taken every $3 \mathrm{~mm}$ and an Intravenous contrast was used. The CT laser beam was used to define the reference isocenter and was placed over the superior orbital ridge. CT images were then transferred to the Eclipse treatment planning system (TPS v8.6).
Table (1): Patients and tumor characteristics.

\begin{tabular}{|c|c|c|}
\hline & No. & $\%$ \\
\hline $\begin{array}{l}\text { Age: } \\
\quad<40 \text { years } \\
\quad>40 \text { years }\end{array}$ & $\begin{array}{l}12 \\
8\end{array}$ & $\begin{array}{l}60 \\
40\end{array}$ \\
\hline $\begin{array}{l}\text { Sex: } \\
\quad \text { Male } \\
\text { Female }\end{array}$ & $\begin{array}{l}7 \\
13\end{array}$ & $\begin{array}{l}35 \\
65\end{array}$ \\
\hline $\begin{array}{l}\text { Performance status: } \\
\quad 1 \\
2 \\
3\end{array}$ & $\begin{array}{l}11 \\
6 \\
3\end{array}$ & $\begin{array}{l}55 \\
30 \\
15\end{array}$ \\
\hline $\begin{array}{l}\text { Clinical presentation: } \\
\text { Convulsions } \\
\text { Diminution of vision } \\
\text { Headache } \\
\text { Weakness }\end{array}$ & $\begin{array}{l}4 \\
1 \\
11 \\
4\end{array}$ & $\begin{array}{l}20 \\
5 \\
55 \\
20\end{array}$ \\
\hline $\begin{array}{l}\text { Pathological type: } \\
\text { Astrocytoma } \\
\text { Oligodendroglioma }\end{array}$ & $\begin{array}{l}18 \\
2\end{array}$ & $\begin{array}{l}90 \\
10\end{array}$ \\
\hline $\begin{array}{l}\text { Pathological grade (WH } \\
\text { I } \\
\text { II }\end{array}$ & $\begin{array}{l}5 \\
15\end{array}$ & $\begin{array}{l}25 \\
75\end{array}$ \\
\hline $\begin{array}{l}\text { Tumor site: } \\
\text { Corpus callosal } \\
\text { Frontal } \\
\text { Temporal } \\
\text { Intraventricular } \\
\text { Thalamic } \\
\text { Parietal } \\
\text { Parieto-occipital } \\
\text { Temporal } \\
\text { Tempero-parietal }\end{array}$ & $\begin{array}{l}2 \\
2 \\
1 \\
2 \\
1 \\
4 \\
2 \\
4 \\
2\end{array}$ & $\begin{array}{l}10 \\
10 \\
5 \\
10 \\
5 \\
20 \\
10 \\
20 \\
10\end{array}$ \\
\hline $\begin{array}{l}\text { Laterality: } \\
\text { Left } \\
\text { Right } \\
\text { Midline }\end{array}$ & $\begin{array}{l}9 \\
3 \\
8\end{array}$ & $\begin{array}{l}45 \\
15 \\
40\end{array}$ \\
\hline
\end{tabular}

\section{3- Target volume definition, dose and fractionation:}

The Gross Target Volume (GTV) was defined as the primary tumor bed defined on the T1weighted pre-operative MRI. The Clinical Target Volume (CTV) was defined as a safety margin of $1.5 \mathrm{~cm}$ in all directions around the GTV to include areas at risk of harboring microscopic disease with manual adjustments to exclude bones and parts of the contra-lateral brain where no invasive tumoral growth has occurred in cases were the tumor didn't cross the midline. The Planning Target Volume (PTV) are generally a $0.5-\mathrm{cm}$ expansion of CTV to account for potential setup errors and patient motion.

Regarding the OAR, manual contouring of the brainstem, cochleae, eye globes, eye lens, optic nerves and chiasm was done. Auto-contouring of the normal brain tissue was performed and the remaining healthy brain tissue volume was delineated automatically by subtracting the PTV from 
the whole brain tissue by a margin of $0.3 \mathrm{~cm}$. Beam energy of $6 \mathrm{MV} \mathrm{X}$-rays was used. The prescribed dose was $54 \mathrm{~Gy} / 30$ Fractions $/ 6$ weeks $(1.8 \mathrm{~Gy} / \mathrm{F})$ to the PTV.

\section{4- Radiotherapy treatment planning:}

All plans were done by the same medical radiation physicist. In the 3D-CRT plans, patients were treated using 2 to 4 static non-coplanar fields while the RA plans were done using 2 half arcs or full arc therapy. The calculations were conducted with the Millennium-120 leaf MLC. Optimization and dose calculations were done using the Anisotropic Analytical Algorithm (AAA) on the Eclipse planning system, Version 8.6.15 (13-14). The plans were calculated with $6 \mathrm{MV}$ photons. Fig. (1) illustrates the number of beams and arcs in pie charts used in the 3D-CRT and RA planning, respectively.

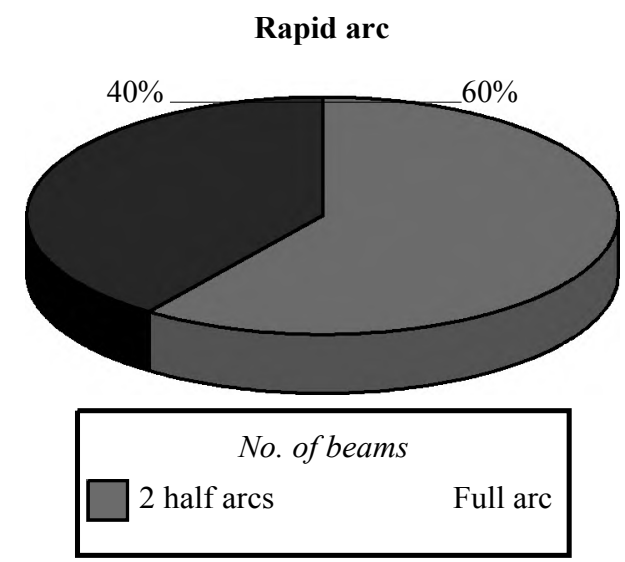

Fig. (1): Number of beams for the 3D-CRT plans (left) and arcs for the RA planning (right).

\section{Plan evaluation:}

A total dose of $54 \mathrm{~Gy}$ was delivered to the PTV in 30 fractions. The plans were normalized to $100 \%$ (54Gy) dose. The coverage of PTV V95\% (volume receiving $95 \%$ of dose) was calculated using the ratio of target volume covered by $95 \%$ isodose line divided by the volume of PTV. Other criteria used to assess PTV coverage were V 107\% (volume receiving $107 \%$ of dose), D98\% (minimum dose within the PTV), D2\% (maximum dose within the PTV), D50\% (D median, which is the absorbed dose received by $50 \%$ of the volume). Conformity Index (CI) was calculated using the following equation: TV/PTV were the TV is the treated volume receiving $98 \%$ of dose and PTV is planning target volume receiving $98 \%$ of dose. Similar to the ICRU 83, the Homogeneity Index (HI) was calculated using the following equation (D2\%D98\%)/D50\%.

For the OAR, maximum doses to the brainstem, optic nerves and chiasma were kept below 54Gy. For the cochleae, the mean dose was restricted to 40Gy. Regarding the eye globes, the aim was to limit the mean dose to below 30Gy. For the eye lenses, the goal was to lower the maximum point dose to less than 10Gy. To assess the dose delivered to the remaining healthy brain tissue; the mean dose, $\mathrm{D} 1 \%$ and maximum dose to the healthy brain were calculated [15]
The Dose-Volume Histogram (DVH) for target coverage and doses received by the OAR were generated. Total number of MUs for each plan was also calculated.

\section{5- Statistical analysis:}

Data were statistically described in terms of range, mean, Standard Deviation (SD), median, frequencies (number of cases) and relative frequencies (percentages) when appropriate. Comparison of quantitative variables between the study groups was done using Mann Whitney U-test for independent samples. A probability value ( $p$-value) less than 0.05 was considered statistically significant. All statistical calculations were done using computer programs Microsoft Excel Version 7 (Microsoft Corporation, NY, USA) and SPSS (Statistical Package for the Social Science; SPSS Inc.,Chicago, IL, USA) statistical program for Microsoft Windows.

\section{Results}

Clinically acceptable 3D-CRT and RA plans were achieved in all twenty cases and approved by a senior radiation oncologist. Tables $(2,3)$ shows an overview of the data obtained from DVH analysis regarding dosimetric outcomes for the PTV coverage and doses received by OAR. Data is reported as mean values \pm Standard Deviation (SD). 
Table (2): Dosimetric outcomes for the PTV.

\begin{tabular}{lllr}
\hline Parameter & 3D-CRT plan & RapidAre plan & $p$-value \\
\hline V95\% & $96.0 \pm 2.6$ & $98.0 \pm 1.9$ & 0.006 \\
V 107\% & $0.02 \pm 0.05$ & $1.18 \pm 2.36$ & 0.030 \\
D98\% (Gy) & $50.42 \pm 2.2$ & $51.64 \pm 1.9$ & 0.013 \\
D2\% (Gy) & $55.8 \pm 2.2$ & $56.5 \pm 1.5$ & 0.369 \\
D50\% (Gy) & $53.5 \pm 1.8$ & $55.8 \pm 2.2$ & 0.030 \\
CI95\% & $1.07 \pm 0.024$ & $1.01 \pm 0.012$ & 0.678 \\
HI & $0.131 \pm 0.112$ & $0.097 \pm 0.033$ & 0.114 \\
MU & $257.6 \pm 16.1$ & $355.6 \pm 44.4$ & 0.001 \\
\hline
\end{tabular}

Table (3): Dosimetric outcomes for the organs at risk.

\begin{tabular}{lllll}
\hline Organ & \multicolumn{1}{c}{ Parameter } & $\begin{array}{c}\text { 3D-CRT } \\
\text { plan }\end{array}$ & $\begin{array}{c}\text { RapidAre } \\
\text { plan }\end{array}$ & $\begin{array}{c}p \text { - } \\
\text { value }\end{array}$ \\
\hline - Brainstem & Max.dose (Gy) & $51.2 \pm 16.9$ & $50.5 \pm 18.1$ & 0.758 \\
- Chiasma & Max.dose (Gy) & $45.5 \pm 17.3$ & $43.2 \pm 19.1$ & 0.127 \\
- Rt optic nerve & Max.dose (Gy) & $37.8 \pm 17.1$ & $32.6 \pm 14.4$ & 0.904 \\
- Lt optic nerve & Max.dose (Gy) & $35.1 \pm 19$ & $31.7 \pm 17.3$ & 0.659 \\
- Rt Eye & Mean dose (Gy) & $18.5 \pm 11.6$ & $16.5 \pm 8.8$ & 0.052 \\
- Lt Eye & Mean dose (Gy) & $16.6 \pm 15.1$ & $13.1 \pm 13.2$ & 0.192 \\
- Rt Cochlea & Mean.dose (Gy) & $10.7 \pm 13.4$ & $5.9 \pm 9.1$ & 0.495 \\
- Lt Cochlea & Mean.dose (Gy) & $16.9 \pm 21.1$ & $8.6 \pm 15.9$ & 0.512 \\
- Rt Lens & Max.dose (Gy) & $2.3 \pm 3.2$ & $7.2 \pm 8.1$ & 0.002 \\
- Lt Lens & Max.dose (Gy) & $1.5 \pm 2.1$ & $8.1 \pm 8.4$ & 0.001 \\
- Remaining & Max.dose (Gy) & $56.2 \pm 2.3$ & $50.1 \pm 3.8$ & 0.001 \\
brain volume & Mean dose (Gy) & $15.2 \pm 4.2$ & $14.7 \pm 3.7$ & 0.081 \\
& D1\% (Gy) & $52.4 \pm 2.9$ & $42.1 \pm 5.1$ & 0.001 \\
\hline
\end{tabular}

Target coverage in terms of V95\% was significantly better in the RA plans with a value of 98.0 \pm 1.9 versus $96.0 \pm 2.6$ for the $3 \mathrm{D}$-CRT plans with a $p$-value of 0.006 . Regarding the target volume hot spots represented by V 107\% (the volume receiving $107 \%$ of dose) it was found to be higher with RA than the 3D-CRT plans with values of $1.18 \pm 2.36$ and $0.02 \pm 0.05$, respectively ( $p$-value of $0.03)$.

Regarding the dose conformity which was described in terms of CI95\%, the RA plans gave a better conformity with a CI95\% of $1.01 \pm 0.012$ compared to $1.07 \pm 0.024$ achieved with the $3 \mathrm{D}$ CRT plans. However, this was not statistically significant ( $p$-value of 0.678). Dose in-homogeneity for PTV described in terms of HI was higher for the 3D-CRT plans with a value of $0.131 \pm 0.112$ compared to $0.097 \pm 0.033$ for the RA plans ( $p$ value of 0.114 ).

The dose received by the OAR in both plans was always below the planned limits (Table 3 ). Overall, the OAR contoured in our study (brainstem, optic nerves, chiasm, eye globes and cochleae) received less dose by RA than by 3D-CRT except for both lenses which received higher doses in the RA plans with a significant $p$-value of 0.002 and 0.001 for the right and left lens, respectively. The remaining health brain tissue which resembles the low dose area was also higher in the 3D-CRT plans as compared to the RA. This was not seen in the Dmean of healthy brain tissue which was nearly equal in both plans. However, the maximum dose and D1\% for the healthy brain tissue was significantly lower in the RA plans when compared to 3D-CRT with a $p$-value of 0.001 for both.

With respect to the average MUs \pm SD needed to deliver a dose of $1.8 \mathrm{~Gy}$ per fraction, it was found to be $257.6 \pm 16.1$ for the 3D-CRT plans as opposed to $355.6 \pm 44.4$ for the RapidArc plans with a statistically significant $p$-value of 0.001 which translates clinically into a shorter treatment time for patients receiving conventional 3D-CRT.

Fig. (2) is an axial CT slice showing the dose distribution for both techniques for the same patient. Fig. (3) illustrates the DVH for PTV coverage and doses received by the OARs for both plans.

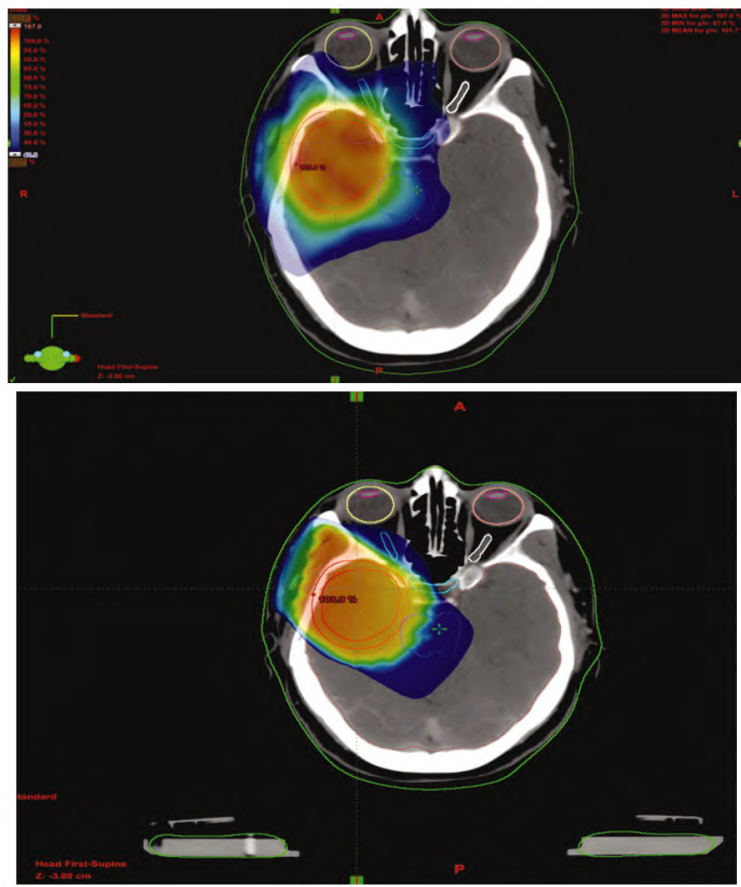

Fig. (2): Dose distribution in an axial view created by doublearc RA plan (top) and a similar CT cut planned by 3DCRT technique (bottom).

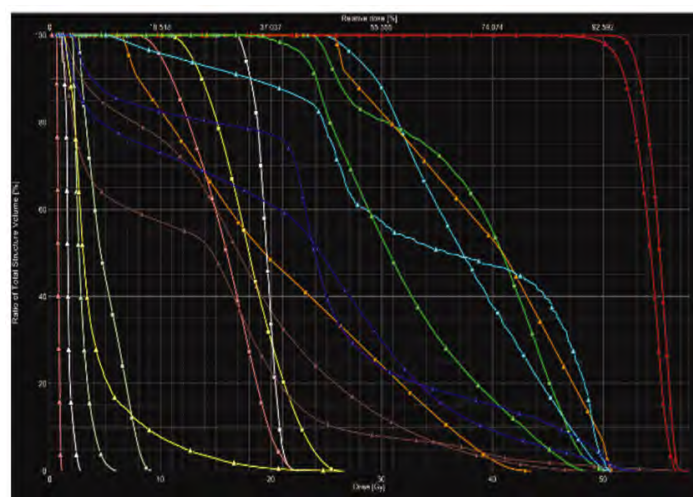

Fig. (3): A Comparative DVHs between 3D-CRT (squares) and double arc RA (triangles). The PTVs are in orange, spinal cord in yellow, brainstem in light blue, the left parotid in purple, right parotid in green and the oral cavity in turquoise. 


\section{Discussion}

There has been an outstanding upgrade in radiotherapy treatment delivery techniques over the past 2 decades. Dose distribution improvements and clinically lower morbidity have been observed with 3D-CRT compared to conventional twodimensional (2D) treatment for different tumor locations [16-18]. Moreover, IMRT has been shown to improve the target volume dose distribution with better sparing of OAR when compared to 3D-CRT technique [19]. Dosimetric studies in different tumor sites comparing RapidArc with IMRT have reported that the plans are comparable but with less MUs and shorter delivery time in the arc delivery [7,12]

Rapid Arc is a novel radiotherapy technology using a complex delivery system achieving beam modulation in a single gantry rotation. Three important parameters are continuously varied to produce IMRT dose distributions-the MLC beam shaping aperture, gantry speed and delivery dose rate [20]. Our study compared RapidArc to conventional 3D conformal technique for patients with low grade gliomas. To our own knowledge this the first study to investigate the potential dosimetric benefits of RapidArc in low grade gliomas as most of the published studies were evaluating the technique in high grade gliomas (WHO GIII-IV).

In a study published by Wagner et al., [15] comparing RapidArc, IMRT and 3D-CRT in patients with malignant gliomas; the authors concluded that if the PTV was distant to OAR, 3D-CRT plans revealed a good PTV coverage of $94.3 \%$ while if PTV was close to an OAR, the 3D-CRT technique showed a poor PTV coverage of $68.2 \%$ compared to IMRT and RapidArc plans. The dose inhomogeneity was higher for 3D-CRT technique than for Rapid Arc and lowest for IMRT. The IMRT plans had a slightly better PTV coverage compared to RapidArc. The RapidArc advantages were less monitor units, shorter treatment time and a small V 107. The RapidArc plans gave the highest D mean of healthy brain while the 3D-CRT plans gave the lowest. On the other hand, the D1\% of healthy brain tissue lowest for Rapid Arc and was highest for 3D-CRT with the IMRT plans lying in between. Regarding the dose to OAR, it was always kept below the constraint limits and was comparable for all three plans. All OAR received a lower dose by Rapid Arc compared to the 3D-CRT and IMRT techniques. The number of monitor units was 1.8 times lower for Rapid Arc than for IMRT and 1.4 times higher than 3D-CRT technique. They found that the irradiation time of Rapid Arc fields was 3.3 times faster than that of IMRT and 1.2 times faster than 3D-CRT technique. The results of our study are in align with the data published by Wagner and his colleagues, the only difference was that in our study the 3D-CRT used a lower number of MUs compared to the RapidArc plans. Similar dosimetric outcomes have been also reported by Goswami et al., [21].

In another study published by Shaffer et al., [22] comparing Volumetric Modulated Radiation Therapy (VMAT) to IMRT for frontal and temporal high grade gliomas, there was almost similar PTV coverage, conformality and homogeneity. VMAT significantly reduced mean and maximum lens, retinal and contralateral optic nerve doses compared to IMRT $(p<0.05)$. Chiasm, Brainstem and ipsilateral optic nerve doses were the same. The mean number of monitor units required to deliver $2 \mathrm{~Gy}$ fractions were $789 \pm 112$ for IMRT and $363 \pm 45$ for the VMAT plans. The authors concluded that VMAT achieved almost equal PTV coverage and OAR sparing compared to IMRT while using less time and fewer monitor units. Having a thorough look at our results, the RapidArc had better sparing of OAR compared to the 3D-CRT plans except for both lenses which received higher doses with the arc treatment. Possibly the reason for this, that we included tumors of different anatomical locations and not just confined to the temporal and frontal lobes as published by Shaffer and his colleagues.

Jun Yuan et al., [23] evaluated the dosimetric differences between IMRT, single (RA1) and double arc (RA2) plans for patients with malignant glioma of the parietal lobe. They concluded that RA1 and RA2 significantly reduced the number of MUs compared to IMRT with slightly better sparing of OAR. Numerous studies has pointed to that the use of two arcs improves the dose distribution compared to single arc plans $[\mathbf{2 4 , 2 5 ]}$. However, based on our radiation physicist experience using 1 full arc or 2 half arcs was sufficient to provide adequate target coverage and lower the dose to the OAR.

Contrasting to the results of other studies, our study showed that the 3D-CRT plans used a lower number of MUs compared to RapidArc reaching statistical significance ( $p$-value of 0.001 ). A possible explanation for this finding is that $70 \%$ of the 3DCRT was performed using 3 fields which had an impact on the overall number of MUs in the conformal plans.

\section{Conclusion:}

At our institution with early RapidArc experience, RA plans dosimetrically achieved a better 
PTV coverage, dose conformity, more homogenous dose distribution and better OAR sparing when compared to conventional 3D-CRT plans except for both lenses. The 3D-CRT plans utilized a lower number of MUs than RA.

\section{References}

1- OSTROM Q.T., GITTLEMAN H., FARAH P., et al. CBTRUS statistical report: Primary brain and central nervous system tumors diagnosed in the United States in 2006-2010. Neuro. Oncol., Nov., 15 Suppl 2: ii1-56, 2013.

2- KALOSHI G., PSIMARAS D., MOKHTARI K., et al.: Supratentorial low grade gliomas older patients. Neurology, 73 (24): 2093-8, 2009.

3- POURATIAN N., ASTHAGIRI A., JAGANNATHAN J., SHAFFREY M.E. and SCHIFF D.: Surgery insight: The role of surgery in the management of low-grade gliomas. Nat. Clin. Pract. Neurol., 3: 628-39, 2007.

4- VAN DEN BENT M.J., AFRA D., De WITTE O., et al.: Long-term efficacy of early vs delayed radiotherapy for low-grade astrocytoma and oligodendroglioma in adults: The EORTC 22845 randomised trial. Lancet., 366: 98590, 2005.

5- BRADA M. and HAYLOCK B.: Is Current Technology Improving Outcomes with Radiation Therapy for Gliomas. Am. Soc. Clin. Oncol. Educ. Book., e89-94, 2014.

6- CHUI C.S., CHAN M.F., SPIROU S., et al.: Delivery of intensity-modulated radiation therapy with a conventional multileaf collimator: Comparison of dynamic and segmental methods. Med. Phys., 28: 2441-9, 2001.

7- VERBAKEL W.F., CUIJPERS J.P., HOFFMANS D., et al.: Volumetric intensity-modulated arc therapy vs. conventional IMRT in head and neck cancer: A comparative planning and dosimetric study. Int. J. Radiat. Oncol. Biol. Phys., 74: 252-9, 2009.

8- YU C.X. and TANG G.: Intensity-modulated arc therapy: Principles, technologies and clinical implementation. (Review). Phys. Med. Biol., 56 (5): R31-54, 2011.

9- BHIDE S.A. and NUTTING C.M.: Advances in radiotherapy for head and neck cancer. (Review) Oral. Oncol., 46 (6): 439-41, 2010.

10- OTTO K.: Volumetric modulated arc therapy: IMRT in a single gantry arc. Med. Phys., 35: 310-7, 2008.

11-LAGERWAARD F.J., MEIJER O.W., VAN DER HOORN E.A., et al.: Volumetric modulated arc radiotherapy for vestibular schwannomas. Int. J. Radiat. Oncol. Biol. Phys., 74: 610-5, 2009.

12- KJAER-KRISTOFFERSEN F., OHLHUES L., MEDIN J., et al.: RapidArc volumetric modulated therapy planning for prostate cancer patients. Acta. Oncol., 48: 227-32, 2009.
13- VAN ESCH A., TILLIKAINEN L., PYYKKONEN J., et al.: Testing of the analytical anisotropic algorithm for photon dose calculation. Med. Phys., 33: 4130-48, 2006.

14- FOGLIATA A., NICOLINI G., VANETTI E., et al.: Dosimetric validation of the anisotropic analytical algorithm for photon dose calculation: Fundamental characterization in water. Phys. Med. Biol., 51: 1421-38, 2006

15- WAGNER D., CHRISTIANSEN H., WOLFF H., et al.: Radiotherapy of malignant gliomas: Comparison of volumetric single arc technique (Rapid Arc), dynamic intensity-modulated technique and 3D conformal technique. Radiotherapy and Oncology, 93: 593-6, 2009.

16-ZELEFSKY M.J., MARION C., FUKS Z., et al.: Improved biochemical disease-free survival of men younger than 60 years with prostate cancer treated with high dose conformal external beam radiotherapy. J. Urol., 170: 1828-32, 2003.

17- LEIBEL S.A., KUTCHER G.J., HARRISON L.B., et al.: Improved dose distributions for 3D conformal boost treatments in carcinoma of the nasopharynx. Int. J. Radiat. Oncol. Biol. Phys., 20: 823-33, 1991.

18- SKWARCHUK M.W., JACKSON A., ZELEFSKY M.J., et al.: Late rectal toxicity after conformal radiotherapy of prostate cancer (I): Multivariate analysis and doseresponse. Int. J. Radiat. Oncol. Biol. Phys., 47: 103-13, 2000 .

19- HUNT M.A., ZELEFSKY M.J., WOLDEN S., et al.: Treatment planning and delivery of intensity-modulated radiation therapy for primary nasopharynx cancer. Int. J. Radiat. Oncol. Biol. Phys., 49: 623-32, 2001.

20- BUSH K., TOWNSON R. and ZAVGORODNI S.: Monte Carlo simulation of RapidArc radiotherapy delivery. Phys. Med. Biol., 53: 359-70, 2008.

21- GOSWAMI B., MITRA S., BANERJEE S., et al.: Rapid Arc: Initial experience in high grade glioma. I.J.R.R., 11 (4): 203-6, 2013.

22- SHAFFER R., NICHOL A.M., VOLLANS E., et al.: A comparison of volumetric modulated arc therapy and conventional intensity-modulated radiotherapy for frontal and temporal high-grade gliomas. Int. J. Radiat. Oncol. Biol. Phys., 76: 1177-84, 2010.

23- JUN YUAN, MINGJUN LEI, ZHEN YANG, et al.: Dosimetric comparison between intensity-modulated radiotherapy and RapidArc with single arc and dual arc for malignant glioma involving the parietal lobe. Mol. Clin. Oncol., Jul., 5 (1): 181-8, 2016.

24- GUCKENBERGER M., RICHTER A., KRIEGER T., WILBERT J., BAIER K. and FLENTJE M.: Is a single arc sufficient in volumetric modulated arc therapy (VMAT) for complex shaped target volumes? Radiother. Oncol., 93: 259-65, 2009.

25- PALMA D.A., VERBAKEL W.F., OTTO K. and SENAN S.: New developments in arc radiation therapy: A review. Cancer Treat. Rev., 36: 393-9, 2010. 


\section{دراسلة مقارنة بين العلاج الإشعاعى ثلاثى الابعاد

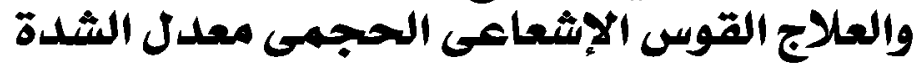

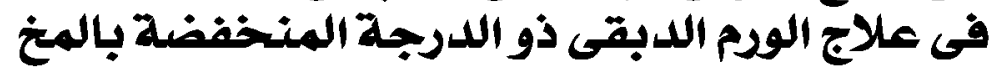

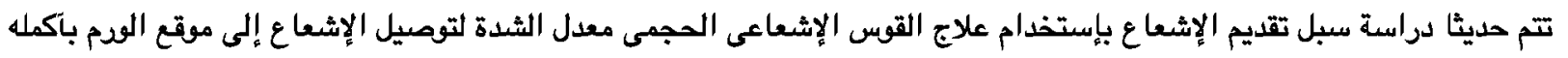

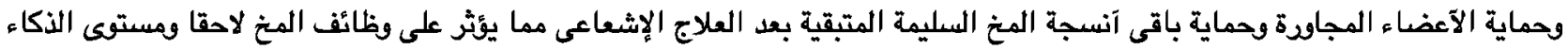

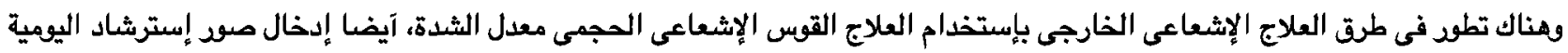

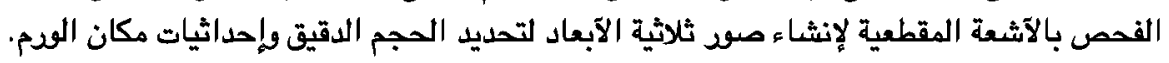

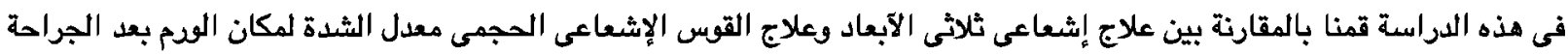

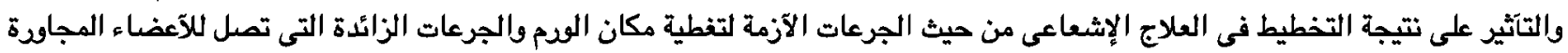

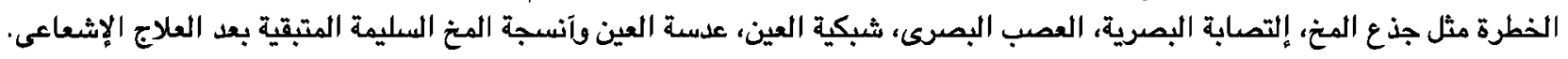

وقد توصلنا إلى وجود إختلاف بينى واضع بفرق إحصائى واضع الجرعات الازمة لتفطية مكان الودم من حيث تفطية آفضل لصالع علاج

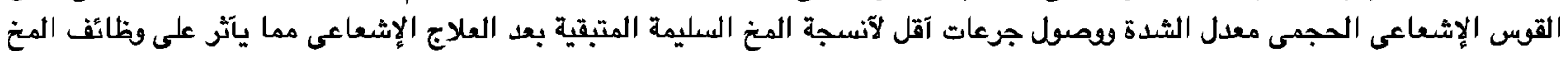
لاحقا ومستوى الذكاء. 